\title{
PHYSICS OF MAGNETIZED ACCRETION DISKS IN AGN
}

\author{
GEORGE FIELD \\ Center for Astrophysics and Osservatorio Astrofisico di Arcetri \\ and \\ ROBERT ROGERS \\ Institute of Geophysics and Planetary Physics, Lawrence Livermore National Laboratory
}

\section{Introduction}

Field and Rogers (1993) proposed that the accretion disks in moderate - $L$ AGN $\left(\lesssim 10^{44} \mathrm{erg} \mathrm{sec}^{-1}\right.$ ) are dominated by magnetic stress rather than gas or radiation pressure. A magnetic field parallel to the disk forms loops above and below it where reconnection accelerates electrons to relativistic energies. The nonthermal radiation observed is the synchrotron emission and inverse-Compton scattering by these electrons.

Most authors have assumed that the magnetic fields are weak, so that $B^{2} / 8 \pi<$ $p$. If they are accreted along with the gas in the dust torus, and if $B$ there is $\sim 10^{-3}$ $\mathrm{G}$, we find that $B^{2} / 8 \pi>p$.

\section{Magnetic Field}

The accretion disk contains matter whose magnetic field $\mathbf{B}_{\mathbf{0}}$ was originally uniform. The flux threading the original region penetrates the accretion disk. In doing so, it is compressed along with the accreting gas, and is wound up by the differential rotation of the disk, forming spiral sectors of alternating polarity separated by neutral sheets. Reconnection of magnetic loops of opposite polarity at neutral sheets limits the strength of the field as it winds up.

At a radius $R$ from the black hole, the radial extension of an adjoining pair of sectors is $\Delta R$. If the local scale height is $H$, the strength of the field along the sector is

$$
\frac{B}{B_{0}}=(2 \pi)^{2 / 3}\left(\frac{\rho}{\rho_{0}}\right)^{2 / 3}\left(\frac{R}{H}\right)^{1 / 3} \frac{R}{\Delta R}
$$

where we have used the conservation of mass and flux starting from $B=B_{0}$ and $\rho=\rho_{0}$.

By balancing the increase in $B$ due to windup with the decrease due to reconnection at speed $v_{M}$, we find that $\Delta R=\left(16 \pi v_{M} / 3 v_{\phi}\right)^{1 / 2} R$. We have shown that $v_{M}<v_{M}^{\prime}$ where $v_{M}^{\prime}$ is the merger speed in the corona, and since according to Petschek (1964) $v_{M}^{\prime} \leq v_{A}^{\prime} / 70$, where $v_{A}^{\prime}$ is the Alfvén speed in the corona, equation 480

T. J.-L. Courvoisier and A. Blecha: Multi-Wavelength Continuum Emission of AGN, 480-481.

(C) 1994 IAU. Printed in the Netherlands. 
(1) implies that

$$
\frac{B}{B_{0}}>7\left(\frac{\rho}{\rho_{0}}\right)^{2 / 3}\left(\frac{R}{H}\right)^{1 / 3}\left(\frac{v_{\phi}}{v_{A}^{\prime}}\right)^{1 / 2}
$$

Plausible values for $\rho, \rho_{0}, H$, and $v_{A}^{\prime}$ yield $B \geq 10^{4} B_{0}$ at the distance from a $10^{8} \mathrm{M}_{\odot}$ black hole inside of which half the luminosity originates. If $B_{0}=10^{-3} \mathrm{G}$, $B>10 \mathrm{G}$, with $B \sim 10^{3} \mathrm{G}$ more likely.

\section{Dynamics}

The equation of motion of a steady-state disk is $\nabla \cdot \mathbf{T}=\rho \mathbf{g}$, where the components of the stress tensor for moving matter plus magnetic field are $T_{i j}=$ $\rho v_{i} v_{j}+B^{2} \delta_{i j} / 8 \pi-B_{i} B_{j} / 4 \pi$. The $R$ component of the equation of motion implies that $v_{\phi}=(G M / R)^{1 / 2}$. The $z$ component gives $\rho=\rho_{*} \exp \left(-z^{2} / H^{2}\right)$ where the scale height is $H=v_{A} R / v_{\phi}$ and where the Alfven speed $v_{A}=B \sqrt{4 \pi \rho}$ is assumed independent of $z$ in the disk. If $\xi \equiv-B_{R} / B_{\phi}$ is independent of $z$, then the $\phi$ component gives

$$
-v_{R}=\xi\left(\frac{v_{A}}{v_{\phi}}\right)^{2}\left[1-\left(\frac{R_{i}}{R}\right)^{1 / 2}\right]^{-1}
$$

Finally the conservation of mass implies that the accretion rate $\dot{M}=-4 \pi \rho H R v_{R}$ $s$ independent of $R$. If $v_{M}$, the merger rate, were known, the above equations snable one to determine $\rho, v_{R}, v_{\phi}, B_{R}$, and $B_{\phi}$ as functions of $R$. If $v_{M}$ is a power aw, all the other variables are also power laws.

\section{Conclusion}

Ve have explored the possibility that AGN accretion disks are magnetically domiated as a result of fields accreted along with interstellar gas. Detailed predictions wait the calculation of the merger rate for coronal magnetic loops.

\section{References}

ield, G.B. \& Rogers, R.D. 1993, ApJ, 403, 94

etschek, H.E. 1964, in AAS-NASA Symposium on the Physics of Solar Flares, (NASA Special Publication SP-50), p. 425 